\title{
The Role of Symbol Form and Legend Design in Proportional Point Symbolised Maps: An Experimental Study in Thematic Map Design
}

\author{
Vineeta John D. Phil \\ Head, Geography Department, Ewing Christian College (An Autonomous Constituent College of Allahabad \\ University), Allahabad
}

\begin{abstract}
Point symbols are the most commonly used proportional symbols in thematic mapping. Traditionally two types of symbols have been in use in this category - proportional squares and circles. These studies have reported malfunctioning of these symbol forms in terms of the errors reflected in response outputs of the magnitudes both in absolute and relative modes when these are read from the symbols placed in map environments. This malfunctioning of the symbol among other factors was ascribed to the type and design of symbol form and the legend provided on these maps. This paper examines the deficiencies in symbol form design of proportional circles and squares together with the existing design practices of legend design. An effort will also be made to improve symbol form by (1) adding (a) uniform gray area fills (b) graded gray area fills in the open circle and square form together with (2) an improvement in legend deign and its organisation. These design improvements will be tested experimentally by measuring the resulting improvements in terms of both efficiency and effectiveness of visual information processing. The experimental results have shown enhancements in both efficiency and effectiveness in information processing from maps due to proposed design improvement.
\end{abstract}

Key Words: Proportional symbolisation, Point symbols, Size variable, Area fills, Legend design Graded gray, Contour, Descriptive rules, Pre-attentive, Focal attentive, Adaptation level, Value variable

\section{INTRODUCTION}

Proportional symbolisation in maps has its strong proponents both in academic and professional circles in thematic cartography. Notable among them are Tobler (1973)[1], Brassel and Utano (1979)[2], Muller (1979)[3] Paterson, (1979)[4]. They take a view that is contrary to the advocates of graded symbolisation and reject the comparison of differences based on the psychological theory of absolute threshold levels on grounds of inappropriateness. The use of discriminable differences of relative levels based on the just noticeable differences, which are much higher (Haber and Hershenson, 1973)[5], is advocated to overcome the difficulty related to the availability of a limited number of discriminable steps. Similarly they have put forward the strategy based on the tendency of human information processing system for chunking the input at the early stages of the human visual information processing, to counteract the arguments based on channel capacity of the human visual information processing system. They strongly argue their case on the grounds of the involved loss of detail particularly, local contrasts. Point symbols are the most commonly used proportional symbols in thematic mapping. The supporters of proportional symbolisation, in case of point, symbols rest their case on these arguments. Their stand in favour of the proportional symbolisation, in addition, has added support in the traditional usage of this symbolisation over a long period of time which still continues to dominate in mapping place data sets.

As for as semantics of the terminology is concerned, the terms Graduated and Proportional, in most cartography texts, are used interchangeably, implying scaling of symbol areas proportionally to absolute attribute values. However, here we will be using Esri terminology where these two terms have specific and different meaning. This is because of the reason of increasing use of GIS software in thematic mapping. The description proportional symbols will be used where scaling of the symbol is done proportional to the absolute data values. The term graduated in Esri terminology refers to the range graded scaling of the symbols. Wright (1944)[6] had first categorised such symbols as point symbols and described these as that kind of symbols which do not correspond with the spatial extent or shapes of the attributes being represented. Traditionally two types of symbols have been in use in this category - proportional or graduated squares and circles.

Proportional circles and the maps symbolised with them are the most common instances of the use of such symbolisation. Though first used during early nineteenth century in 1837 by Henry Drury Harness (Robinson 1955)[7], the use of circles came into wide spread use in the beginning of the $20^{\text {th }}$ century. Flannery's (1956) [8] survey which covered professional journals in geography and the books ranked this symbolisation at the fourth place, with a share of $10 \%$ among the published maps. Another survey (Akhtar 1990)[9] done relatively sometimes back 
points to almost similar results showing that the symbolisation continues to hold the same popularity with the map makers. For the purpose of graphic display of the numerical magnitudes of a distribution at positions in geospace, measured on either interval or ratio scales, the referent magnitudes are converted into another semantic relationship by translating these magnitudes into proportional areal sizes of the circle symbols where:

$$
x_{i=\pi r^{2}}
$$

in which $x_{i}$ is the referent magnitude, $\pi$ is a constant, and $r$ is the radius of the circle symbol. The size of proportional squares takes the form of:

$$
x_{i=a^{2}}
$$

in which the $x_{i}$ is the referent magnitude and $a$ is the length of any side of the square symbol. The area of each such symbol is reduced to a common scaling factor in relation to the range of given referent magnitudes. The areal size of the symbols thus becomes the operative graphic dimension which links the symbolic expression with a graphic form in meaning with the referent content or magnitude.

The semantic relations between the proportional symbols and the referent quantities in data sets when given a graphic expression in map space are built through the descriptive rules which are stated in legends on the maps. These expressions in the form of proportional symbol simultaneously designate the referent contents by indication of referent locations as well by the portrayal of the magnitudes located at these places. This representation of magnitudes involves the use of a form of measurement unit different from the units used to measure the referent phenomena. This is done through the translation of referent one dimensional quantities into the areal size of a two dimensional graphic plane form in such representations. The physical extent of the plan form i.e. the area of the regular geometrical figure of circles or squares operates as one of the aspects of the graphic variable of size in representing the referent contents.

This form of symbolisation and particularly the proportional circle symbolised maps have attracted the attention of quite a large number of cartographers working in the area which is now commonly described as design research in thematic cartography (Flannery, 1956 [8] Williams, 1958 [10]). This trend had started with the doctoral dissertation of Flannery submitted at the Wisconsin University in 1956 and is still continuing. Till few years back these studies were mostly related to the semantic aspect of map symbolisation and were mostly concerned with the retrieval of symbolised magnitude information either from the symbols outside map environment or from proportional circle maps. These studies were limited to $S$ - $R$ relationships in psychophysical tradition of research. These studies have reported malfunctioning of these symbol forms in terms of the errors reflected in responses outputs of the magnitudes both in absolute and relative modes when these are read from the symbols placed in map environments and the symbols used elsewhere outside of the map environments. This malfunctioning of the symbol among other factors was ascribed to the type and design of symbol form ( Wood 1972[11], John 2003[12]) and the legend provided on these maps, the major works which can be cited in this regard were by Meihoefer (1969)[13], Baird (1970)[14], Dobson (1974 )[15], Stevens (1975)[16], Cox (1973[17], 1976[18]), and Chang (1980) [19].

\section{OBJECTIVES}

This paper will examine the deficiencies in symbol form design of proportional circles and squares together with the existing design practices of legend organisations, their role and the resultant design shortcomings vis-à-vis visual information processing from these maps. An effort will also be made to improve symbol form by (1) adding (a) uniform gray area fills (b) graded gray area fills in the open circle and square form together with (2) an improvement in legend deign and its organisation. These design improvements will be tested experimentally by measuring the resulting improvements in terms of both efficiency and effectiveness of visual information processing at preattentive stage of cognitive visual information processing where improvements in processing are most amenable to design improvements.

Visual search will be employed here to test the proposed hypotheses in this respect. Visual search or scanning tasks have largely been treated as investigations into different stages of human information processing. The temporal aspects of the tasks performed at different stages are commonly designated as reaction times or search times and serve as the dependent variable in such investigations. These reaction times have been used as powerful tools in cognitive research when cognition is viewed as a series of processing events taking place over time. Posner and Rogers (1978)[20] have pointed out to the use of reaction times in identifying independent subsystems within the human processing system. In this continuation they have also referred to their utility in distinguishing automatic from attended processes.

\section{PREVALENT SYMBOL DESIGN}

In case of the proportional circle and square symbolisation the symbols take forms that are produced by the black lines which are used to construct the outlines of the circle and square symbols. These provide graphic forms to the referent contents (Fig.1). The area inside these symbols is generally not differentiated from the 
areas lying outside in the focal realm of the map. The only difference is provided the by the outlines of the circles or squares that provide the contours* to symbol shapes. The areas inside of these symbol outlines appear to belong to the same homogeneous plane which is formed by the paper surface in the focal realm of the maps. This homogeneity, in some cases, is broken by the lines symbolising the map's locational input. Only on a very small percentage of these maps, the enclosed space in the circles or squares is filled either with solid black or

A.

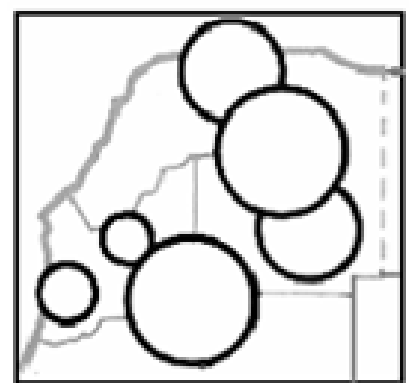

B.

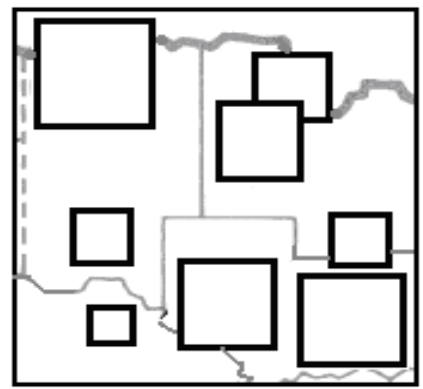

Figure 1: Conventionally used symbol design: (A) proportional open circles and (B) proportional open squares

uniform gray tone. The visual information processes are considerably impaired by the symbol form which has a white area inside the symbol outlines that is indistinguishable from the surrounding surface of the focal realm of the map in proportional symbol maps (John 2003)[12].

\section{LEGEND DESIGN IN PRACTICE}

A survey undertaken to analyse the legend design of this category of maps has revealed that no system or convention is followed for organisation and display of the system of descriptive rules for this kind of symbolisation in the legends. The selection of size of content units, to be graphically displayed as standard legend symbols, on such maps are chosen arbitrarily. Selected content unit values are generally chosen in an arithmetic progression with arbitrarily chosen distances and are chosen in multiples of 5,10 or 100s depending on the magnitudes found in the referent distributions. Only in case of the referent distribution with large ranges, the chosen values are often selected in a geometric progression. But in both of these cases the selected values do not have any kind of direct relation in any way with the magnitudes found in the referent distributions. Same is the case with the number of standard symbols used in the legends. The choice in these, cases is again arbitrary and has nothing to do either with the nature or the range of the mapped referent contents. Table 1 summarises the situation in case of the number of standard symbols found in the legends of these maps and the frequency of their use.

The manner of the organisation of the standard symbol and their display appears to have almost been conventionalised in two forms. These are (1) nested arrangements and (2) strung out arrangements Figures 2.A and 2.B). The frequency of the use of these two arrangements has been found to be almost equal in which the strung out arrangements, when vertical and horizontal arrangements are considered together, has a lead of 5 per cent. As compared to both of these arrangements the frequency of the use of nested arrangement of the standard symbols on these maps was found to be 47 and 40 per cent in circles and squares respectively. In case of the

Table. 1. Number of symbols used in the legends

\begin{tabular}{|cc|}
$\begin{array}{l}\text { Number of } \\
\text { Standard } \\
\text { Symbols use }\end{array}$ & $\begin{array}{l}\text { Frequency of } \\
\text { Use in Percent }\end{array}$ \\
\hline $2-3$ & 24 \\
\hline $\mathbf{4 - 5}$ & 49 \\
\hline $\mathbf{6}-\mathbf{7}$ & 22 \\
\hline$>\mathbf{7}$ & 05 \\
\hline
\end{tabular}

strung out arrangement the vertical ordering was found to be more favoured. Its use in comparison was seen to be 43 and 46 per cent. In all these arrangements the form of the standard symbol was made to appear almost

*The word 'contour' apart from its use in topographic cartography is defined in usages related to human vision in psychology and artificial vision is treated as a sensation which is 'not a physical object in the visual field, but is the place where the perceived figure appears to end'(Dent,1972)[21]. According to Rubin (1958)[22] 'In effect, the common contour indicates this is the end of the object, and seems to concern the ground to such a small degree that the ground can quietly continue on past behind the figure'. 
exactly the same as they appear on the maps. Only in case of some maps using nested arrangements of standard symbols the form of these symbols was not made similar to the symbols used on the maps. In such

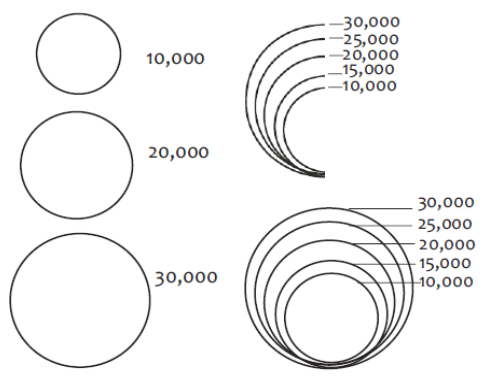

Figure 2.A Conventional organisation of legends in proportional circle maps

cases only half of the circle perimeters in nested arrangement were shown in the legend. This practice however, was mostly found limited to the proportional circle symbolised maps only.

\section{IV.1 The Role of Legends in Conventionally Designed Maps}

The proportional symbolisation requires a code or descriptive rules for linking the referent contents and their graphic expressions in semantic relations. Unlike the semiotic system of natural languages the semiotic
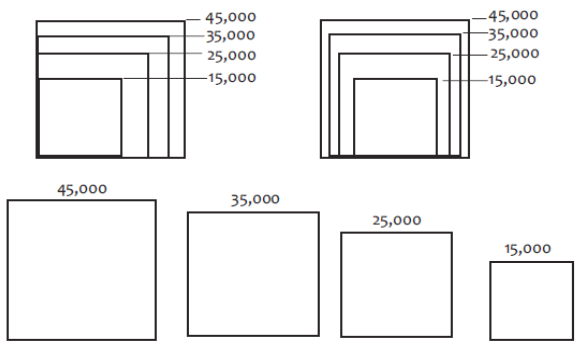

Figure 2.B Conventional organisation of legends in proportional square symbolised maps

system of map graphics involves a system in the form of legends to establish such a linking code or descriptive rules (Schlichtmann 1979 [23] and 1985 [24]) to assign meanings to map symbols. The legends, in such instances, act as the organising systems for the established codes. The role of descriptive rules and the system where these rules are organised assumes greater significance on thematic maps because of the fact that units of map language are more than often redefined from representation to representation on different maps with different referent distributions.

The legends used on these maps which relate the proportional point symbols with their referent contents function differently from some other forms of symbolisations. The nature of these links of meanings as well the manner of their display and use are quite different from other symbolisations, particularly, proportional symbolisation. It does not link the sign vehicles with their referent contents directly as is the case with the legends of maps using range-graded symbolisation. The legend as the system which is used to organise the descriptive rules that link the sign vehicles with referent contents in relations of meaning, assumes more importance in case of these maps. This is because of the fact that the links of meanings between the proportional symbols and their referent contents are not structured directly in case of these maps.

The links of meaning, in this symbolisation are provided indirectly through few standard symbols in the legends. In view of the organisation of the linking codes on these maps, the semantic relations between the proportional symbols displayed on the map and the referent contents, in terms of their numerical magnitudes, are determined through the use of these standard symbols in the legend. Any of these standard symbols can be treated as an anchor and the magnitudes of the displayed referent contents of a distribution by these symbols are then estimated by relatively comparing it with the selected anchor.

\section{SYMBOL FORM, LEGEND DESIGN, AND VISUAL INFORMATION PROCESSING}

The experimental evaluation of the efficiency and effectiveness of the proposed map design will be done taking into account the two-staged hierarchical human visual information processing generally named as 'preattentive' and 'focal attentive'(Neisser, U. 1967 [25] Treisman, A. and Gelade, G. 1980 [26], and Wolfe, J. M. 1994) [27]. The processes of the first stage in the system are taken to be carried out in parallel very early in the processing and are very fast. These processes segment the visual input to the system into the figure and 
ground components. Subsequently the input is taken to be segregated into chunks that are organised in hierarchically arranged vertical planes along the dimension of depth. Such organisations are particular to processing of two dimensional images or scenes; and are primarily based on relations of similarity, texture and density gradients, surface contrast gradients which include gradients of brightness or value. The processes at the second level involve deployment of visual attention and serial processing. These processes are seen to be engaged with some kind of constructive activities, which synthesise and build the visual figure from the preattentively processed input. In case of map images such synthesis may be seen to result in the comprehension of displayed contents through symbol discrimination, meaningful identification of symbols and symbol configurations as the constituent structure of the map image.

These two processes of visual information processing operate through the complementary 'bottom -up' and 'top-down' mechanisms. Palmer (1975)[28] while elaborating this convergent and simultaneous sequence of operations suggests that in the initial phases of information processing from a scene or image display (such as maps) operations of segregation and feature extraction are performed on the input data by bottom-up mechanisms. Relying on Eastman's (1985)[29] extension of Navon's (1977)[30] findings to other visual displays, it can be surmised that, in case of maps too, the convergent operations of the visual information processing mechanisms begin with the broadest global properties of map image and then move incrementally to local features or finer details which builds up the map image and is seen to lead to map comprehension in successive stages.

\section{V.1 Symbol Discrimination}

Discrimination of map symbols depends on ordinal level size information available from sensory information on size which structures the relations of order in the later phases of preattentive processing. Such relations of order are produced due to the presence of gradients in the visual input. Gradients are defined by Arnheim (1969:268)[31] 'as the gradual increase or decrease of some perceptual quality in space.' These perceptual gradients are organised along the dimension of depth or distance (Gibson 1950)[32] which produce an organisational hierarchy of symbol sizes in which the largest symbols, representing the largest magnitude, lie on a plane nearest to the map percipient while the smaller symbols in order of their size move to successively lower visual planes.

Arnheim (1969:268-269)[31] in this respect further points out the requirements of obliquity in positional arrangement, regularity in order and spatial intervals in distributional patterns to enhance the perception of depth. These are not possible to be met in the present instance of symbolisation and mapping due to the nature of the displayed information in which the arrangement of the circle symbols on the map is controlled by the facts of the location of data and where these locational facts cannot be rearranged through any deliberate design effort. Whatever obliquity of relative locations is available is unintended and is inherent to a particular mapped distribution. The limitation of data locations at the same time makes it impossible to obtain consistently decreasing spacing between symbol locations on the maps. In view of this, the factor related to consistent decrease in spatial intervals between symbols cannot be possibly met here. In addition, the magnitude differences in a distribution and the differences portrayed in proportional characterisations may not always be large enough to be seen as conspicuous differences of symbol sizes. Due to these reasons the symbol sizes characterising the magnitude intervals in a distribution may or may not always exhibit a gradual decrease.

Moreover, in case of conventional proportional symbolisation with open symbols, fulfilling of the requirement of perceptual decrease in symbol size, is subject to the control of the number of discriminable steps available in case of the graphic variable of size in the form of areal size of circles or squares. In addition to this, the graphic appearance of these symbols imposes constraints on the availability of information on areal extents of such symbols to the system from the maps (Wood 1972) [11]. As a result, operationally parallel global analysers may not be able to extract adequate information on the areal size of these proportional symbols and may fail to make areal size based differentiations effectively. This can take place even when the structured differences happen to be larger than the required JNDs. In view of these two factors the information on obvious and gradual decrease of symbol size may not be available to the system due to which the engendered size gradient may lack the required effectiveness (John 2003) [12].

The ambiguities in available areal size information and the resulting size based discriminations of the open square or circle symbols, in addition to their effects on size gradients, extend to produce multistability in the hierarchical organisation of segregated chunks. The organisation of similarity linked groups based on such information may not result in the simultaneous assignment of each such group to its respective plane in the third dimension of depth. The possibility of location of such equivocal chunks in alternate planes becomes equally good causing this kind of multistability. The map percipient in such cases might experience a sudden and involuntary change in the apparent position of a symbol or group of symbol along the depth dimension. Another kind of multistability may also arise in the three dimensional organisation due to the alternations in the attribution of surface properties to the different parts of the map image. In such cases the symbols, in one 
instance, will tend to be organised in more than one visual level. In the other case, as seen in case of figure ground segmentation, the map image may be seen as a white surface through which different sized circles have been cut out. As a result the image may not at all be organised in the third dimension as required (John 2003) [12].

\section{PROPOSED DESIGN IMPROVEMENTS}

The use of area fills of both uniform gray and graded gray and the other involved design changes, will apply to different steps in the structuring of the map image, particularly, the thematic component of the map and the related organisation of the descriptive rules in the legend. The change will have to address itself to all those aspects of the maps that were identified for some kind of deficiency and where the use of area fills in open circles and squares might result in increasing the efficiency of the visual information processing system through an enhancement of the compatibility of the maps with the human visual information processing system.

\section{VI.1 Proposed Symbol Design}

The semantic relations between the proportional symbols both circles or squares and the referent magnitudes in the proposed design are basically based on the areal size of symbols. Grey area fills both uniform and graded are used in a configural redundant mode. In the first case the grey area fills were used to do away with the inadequacy in both symbols produced due to symbol form defined by the outlines of the symbol only (Fig.3). At the level of structuring the symbol form this change will relate to the physical appearance of the sign vehicles.

In the second case of area fills of graded gray an additional graphic variable of 'value' was added to the size variable redundantly. The mode of symbolisation will take a form in which the simultaneous and joint modulations of both the variables will correspond with the variations in the referent magnitudes. The operative dimension in such characterisation will not be size alone but will be defined jointly by the size and a value step in gray scale (Figure 4). The structuring of the symbol with this change will reflect certain aspects of the

A.

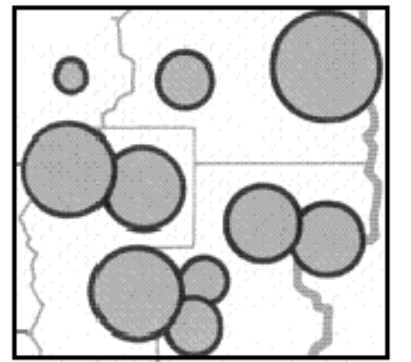

B.

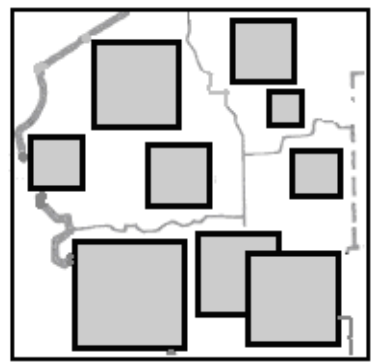

Figure 3. Proportional circle symbol forms (A) and proportional square symbol forms (B) with uniform gray area fills

physical appearance of the sign vehicles. The areal extent of the proportional symbols thus is intended to take on much of the graphic appearance of area symbols.

A.

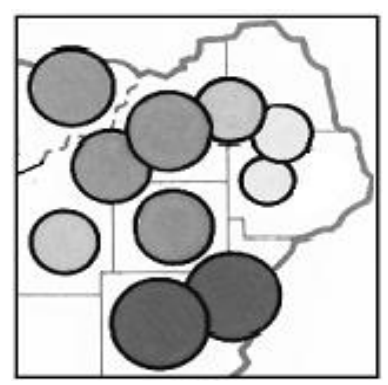

B.

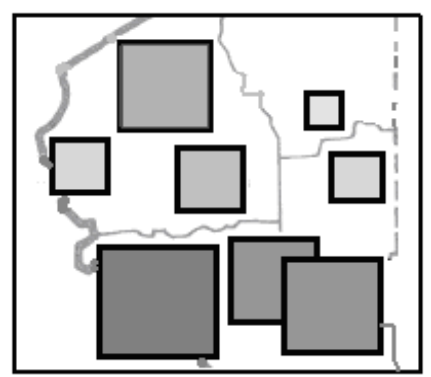

Figure 4. Proportional circle symbol forms (A) and proportional square symbol forms (B) with graded gray area fills

The resultant appearance relates to the surface character of the areas enclosed by the black symbol outlines in both instances of design improvement, which were left undifferentiated in most of the cases earlier. This surface character emerges from the efforts to differentiate the enclosed symbol areas from the plane surface are of focal realm by the graphic application of uniform as well graded gray area fills within proportional symbol outline forms. 


\section{VI.2 Proposed Legend Design: Content, Structure and Organisation}

The next step where this improvement in design will apply is concerned with the way in which the links of meaning between the map symbol form and referent contents are structured; and the manner of the organisation of this linking code in the form of descriptive rules in the legend in both cases. The functional role of legends in the visual processing, when viewed in terms of efficiency in information processing together with the cognitive/semiotic approach, points towards certain inadequacies that should be met in the legend design of these maps. This need has to be taken into account if it is desired to maintain functional efficiency of the human visual information processing system for obtaining effective response outputs from these maps in terms of ease and accuracy as well improving their usability.

One of the first such aspects of the requirements in the design improvement is available in the views of Robinson et. al.[33] that relate to adhering to the required practice of making the standard symbols in the legend appear "exactly as they look on the map drawn in precisely the same - - - manner" (Robinson et. al., 1995:336) [33]. The other such aspect in this regard can be seen in the experimental evidence from efforts aimed at legend design improvements (DeLucia and Hiller 1982) [34]. Their results can be extended to show that those legend designs function more efficiently where the arrangement of the standard symbols follows the same kind of ordering which was employed during the modulations of the required graphic variable or variables for attaching a form to the referent attributes at their various designated places in the map space. Put in other words the effective standard symbol arrangement in the legend should simulate the arrangement of visual planes of different data magnitudes built in the map space along the $z$ dimension of the map (Fig.5).

The next such consideration relates to the optimal number of standard symbols and the size of content units to be displayed as standard symbols in the legends. This can be achieved by selecting the optimal number of classes and their sizes from the number of different sizes of referent contents or the number of the occurrences of different data values in a given data set for an area. Another requirement to be met is that the selected classes should be chosen by such breaks in the referent distribution which divides the data array into unambiguous and mutually exclusive groups and the resultant data classes or intervals must meet the criteria of minimum within group variance and maximum between group variance as required in such data classifications.

Another most important requirement in this continuation relates to the size of content units to be displayed in the legends as standard symbols for a given referent distribution. Chang's (1969)[35] and Cox's (1973 [17], 1975[36] and 1976[18]) demonstration of the advantages of using Helson's (1953)[37] adaptation level theory points to a solution of this problem. A selected central tendency measure in the form of the geometric mean in the form $\log G m=1 / N \sum \log X_{i}$ which describes the selected classes can be chosen as the content magnitudes for their graphic transformation into standard symbols in the legend. The number of such

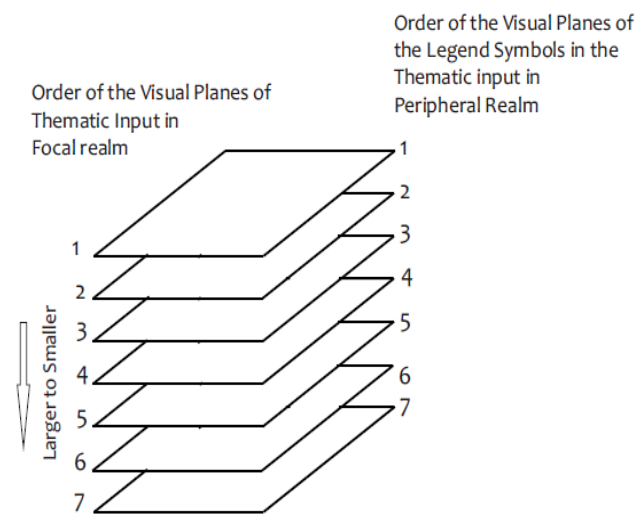

Figure 5. Designed organisation of visual planes in the $z$ dimension of the map symbols and the required organisation

standard symbols, in view Of the nature of the class intervals and their exhaustive coverage of the referent distribution domain, are expected to meet the adequacy criteria set for this purpose.

Apart from these two aspects of the legend design there is an equally important inadequacy is that there is no provision in the design through which the attention of the map percipient be directed towards the right standard symbol in the legend and that the map percipients be able to use it as an anchor in any symbol discrimination event. The form of these symbols is required to have some graphic properties through which they could be accessed through bottom-up mechanisms when ever required for top down verification during processing of information from these maps. These form qualities arising out of these graphic properties can be summarised as specificity, category membership cues and memorability. These form qualities are needed for 
engendering the relations of association between the target and the required anchor symbols in the legend. These form qualities (Baird 1970 [14], Stevens 1975 [16]) are sought to be achieved through the proposed symbol design change by using graded gray area fills in place of uniform area fills and their use in the design of the standard symbols too (Fig.6).

\section{VI.2 Layout and Organisation of Graphic Components of Map Image}

This step, in the map image structuring, too reflects an extension of the efforts at increasing the compatibility of the map image with human information processing system. Towards this end the suggested design, in addition to its major proposals, attempts to incorporate the relevant design principles that have already been developed in this area (Wood 1968 [38], Dent 1972 [21], Robinson et al. 1995 [33], Cuff and Matson
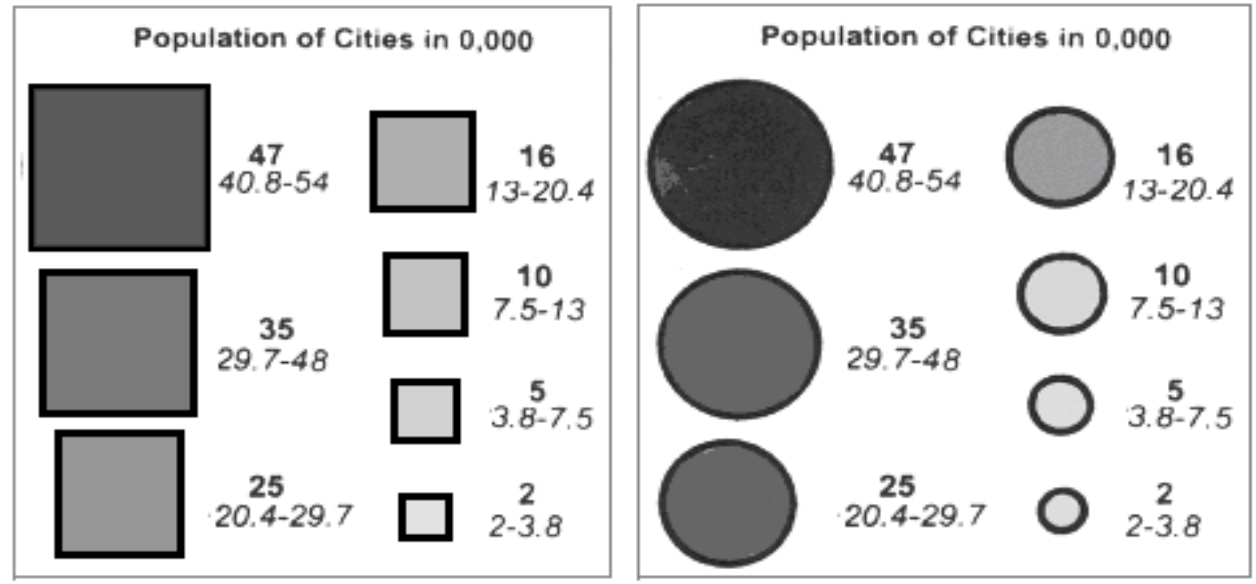

Figure 6. Prototypes of improved legend design and arrangement in proportional circle and square symbolised maps. Bold figures denote class geometric means used as radii for standard symbols in legends. Figures in italics show the class limits.

1984[39]). This exercise takes into account the fact that the map image is composed of substantive components with varying significance and different functional roles. These components are to be organised in terms of the spatial order necessary to relate the information carried by these components to one another in order to create a coherent map form.

This kind of image component ordering has been used to establish the narrative of the graphic statement of the map or in other words to organise the contents of map text. Use of graphic characteristics has been suggested for this kind of organisation also (Dent 1972 [21], Castner 1983) [40]. Building upon Dent's work Castner (1983 p.106 [40]), particularly, advocates modulations of graphic contrast to achieve the desired ordering. Such organisations too are made along the dimension of depth in which the substrate forming the primary ground of the image is relegated to the lowest most visual level. The different map components then are arranged in a nested hierarchy of figures of varying functional and visual importance. The map image for the purpose of this organisation was first divided spatially following Cuff and Mattson (1984) [39] into two visual realms. These have been named as peripheral and focal realms according to their location and functional roles. At the second level the information components in these two realms have been classified into information categories of locational and thematic components.

The locational components in both realms have been subdued to minimum legibility levels necessary for their access to focal attention when desired during processing of thematic components or recognition of the spatial relations in the focal realm. The thematic component in the focal realm has been kept at the upper most level in this ordering through strong surface contrasts and high degree of heterogeneity. Similarly different units of thematic components, particularly the legend, belonging to the peripheral realm too were separated and organised in a way that brought them to the visual level of the thematic input (Dent 1972) [21]. The other elements too were organised in a manner that arranges these at a higher visual level than the locational component in this realm. This ordering was accomplished by the graphic contrast produced by the black lettering against the gray elements of the locational input. Contrasts of gray and heterogeneity in the figural field were used to separate legend from the locational information of the peripheral realm. The use of gray area fills both uniform and graded and the proposed arrangement of legend symbols in this design create a problem at this stage. The legend in this design, because of the similarity of its visual level with the thematic component in the focal realm and its arrangement may appear as an extension of the focal realm itself. This extension effect was eliminated by the spatial separation of the legend from the focal realm 
following Dent (1972) [21] by putting the legend in a box of black or gray out line and superimposing it over the outline of subject area (Figures7, 8 and 9).

\section{EXPERIMENTAL EVALUATION}

The experimental evidence in cognitive visual information processing and experimental results in design research in cartography provide grounds for expectancies that the improvement in proportional symbol form deficiencies will increase the functional efficiency and effectiveness of such symbols.

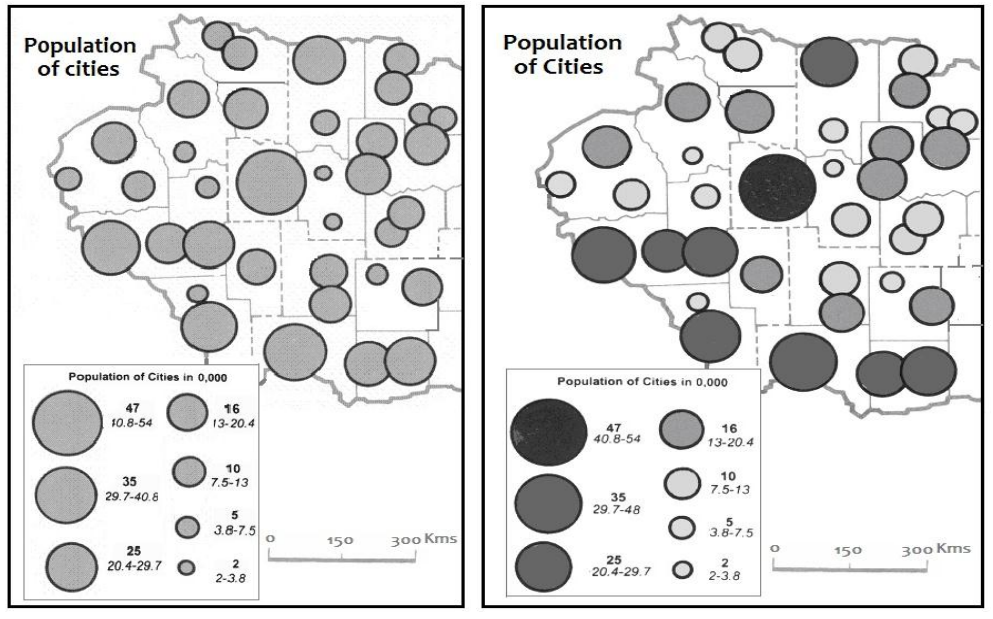

Figure 7. Prototypes of improved map design of proportional circle symbolised maps

Following these expectancies the research question in the experimental evaluation was related to symbol size discriminations of represented by the symbols. The research question in this respect was posed as:

'whether the improvement in traditional outline proportional symbol form by providing area fills of uniform grey and/or graded grey with the legend design improvement will lead to increase the functional efficiency and effectiveness of such maps?'

The hypotheses tested to answer the question took the following forms.

'The improvement in traditional outline proportional symbol forms of circles and squares, by providing area fills of uniform grey and/or graded grey with the legend design improvement, will result in an increase in the functional efficiency and effectiveness of such symbols in symbol size discriminations'.

The experimental evaluation of functional efficiency and effectiveness of the proposed design improvements will be made by the use of the following two measures: (a) the efficiency that will be seen in terms of ease with which the task is performed as measured by the processing speed i.e. the reaction time or the time taken in processing the information related to a specified task; and (b) the effectiveness as ascertained by the accuracy of the responses which will be determined by comparison of response outputs with the mapped information

\section{VII.1 Experimental Method}

Fifty subjects, drawn from the University of Allahabad and its constituent colleges were used in the experiments. The subjects comprised of both genders. The subjects were taught about proportion symbol mapping and were expected to have used such maps during their studies. The experiments in this study were made on the equipment designed by modifying the circuitry of a reaction time measuring digital chronoscope capable of recording time in milliseconds.

Experiments were developed to test the hypotheses of efficiency and effectiveness of performance related to the symbol size discriminations. Visual search has been used to test the hypothesis for symbol discrimination. Reaction or processing times for different interdependent tasks or stages of visual information processing from the maps have been obtained through the revision of Donders' (1868)[41] subtraction method in certain important respects. Such a revision appears to have been first attempted by Neisser (1963 [42], 1964)[43] in his character recognition experiments. Results of this analysis were later used by Neisser in support of his two-stage model of human visual information processing. The attempts at revisions of Donders' 
subtractive method were put on a firm methodological ground by Sternberg through a series of papers (1966[44], 1967[45] and 1969[46]).

The revision in the experimental method primarily relates to the assumption of pure insertions in Donders' [41] method, which have often been found invalid. This is in addition to operational difficulties associated with such experimental manipulations. The revised method does not require either insertions or deletion of any information processing tasks or stage through some experimental manipulation. In the revised method, instead of inserting such a task through experimental manipulation for measuring the duration of a
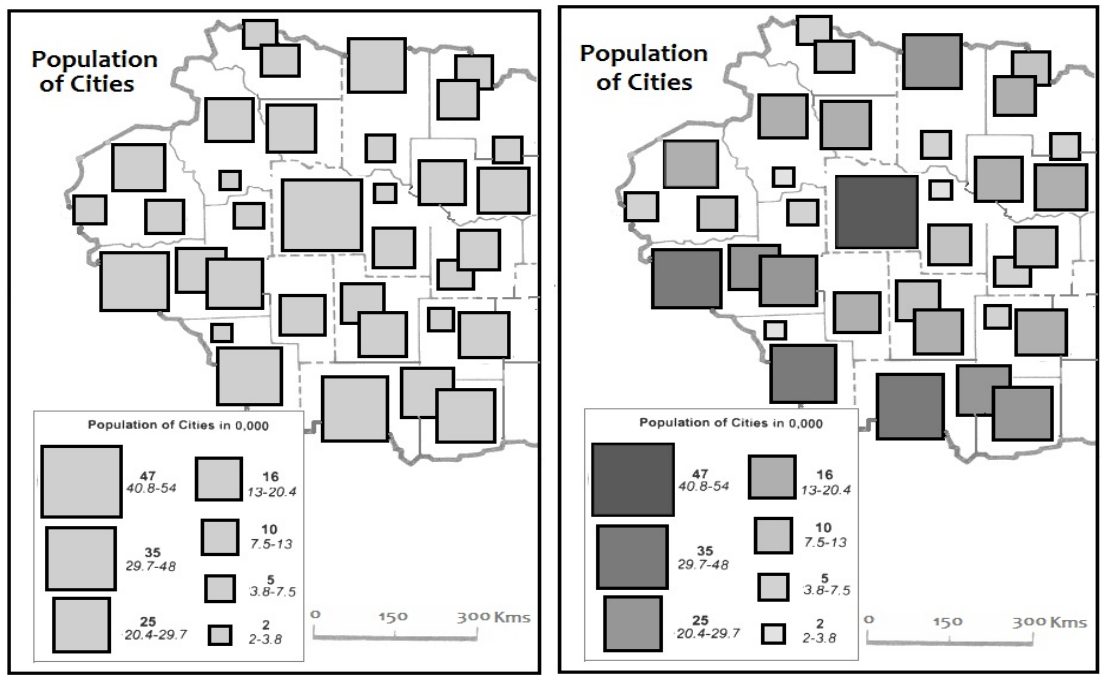

Figure 8. Prototypes of improved map design of proportional square symbolised maps

selected such task or stage, the duration is measured by experimental manipulation for varying ' $n$ ' the number of times the task in question operates. Similarly, the revised method does not require experimental manipulations through which the task of interest is deleted. In its place, the deletion of either the task or stage in question is obtained by extrapolation and the duration of other interdependent tasks as well stages are obtained mathematically without or in absence of a component task or stage.

This method of obtaining separate measures of reaction times for different stages or tasks in visual information processing is based on the principles involved in the two methods namely the subtractive method and the additive factor method. The use of subtractive method depends on the experimental manipulation of varying ' $n$ ' under the assumption that if the time to visually process ' $n$ ' items is subtracted from the time spent on processing $n+1$ items, the difference between the two is an estimate of the time taken for the visual processing of a single item. This search rate per item, in practice, is obtained as average visual processing rate per item by fitting a straight-line function to the data by the method of least squares. The fitted function takes the form of $y_{c}=a+b x$. The regression coefficient ' $b$ ' or the slope of the fitted line is a measure of average processing rate per symbol of a given category.

However, the validity of this subtractive technique rests on two assumptions. The first such assumption concerns itself with the nature of the visual processing. Under this assumption the tasks should be exhaustive in nature and not self-terminating. The second assumption relates to the linearity of the fitted function. The linearity is suggestive of the fact that the time between onset of the input and response output is occupied in part by a serial comparison, classification, and identification processes. The encoded representation of the standard proportional symbol is compared successively with each symbol in the target areas. The linearity further implies that the time spent or needed for each symbol in each such task is almost equal and does not vary from symbol to symbol.

Similarly the time spent on other such tasks stages, when the intended task in processing is not operating theoretically, is obtained from the ' $y$ ' intercept of the fitted regression function as stated earlier. This manipulation of the data for obtaining the processing time in the absence of a designated task without actually deleting it depends on the additivity of the processing interdependent tasks or stages. This additivity implies that the time span of every such single task contributes individually to the total processing time. In this way the obtained total reaction or processing time is the sum of the time taken by each such separate task or stage.

\section{VII.2 Symbol Discrimination}

Visual search will basically involve an ordinal level size based symbol discrimination and classification task at the ordinal level. This task of discrimination will be performed indirectly. The subjects will 
be required to search and find the location of proportional symbols, which are equal in size to the provided circle or square symbol while performing the following task:

"A State government's health ministry has to improve health care facilities in each of the cities of the region marked on the map. In this effort the availability of doctors, nurses and beds in hospitals will be determined in proportion to the size of the population of the cities. A city of one size class has been identified for such determination at some sample locations. Find out other cities, which have the same size of population in the area."

During this kind of scanning the subjects will be comparing the symbols in the target area with the selected circle or square provided for this purpose and in the process will be performing discrimination and classification at ordinal level. The proportional circle or square symbols found either larger or smaller in size as compared to the provided symbol during the scanning will be rejected as mismatches and will elicit no response.

The requirement of the revised method for experimentally varying ' $n$ ', the number of times the processing stage in question is supposed to operate, will be met by selecting the target areas with varying number of proportional symbols. The assumptions related to an exhaustive search, necessary for use of this revised version of subtractive method, has been met by designing search task in a way which will exclude the possibility of the termination of the search when a target is located. The search will continue till every symbol is compared with the target symbol making the process exhaustive.

The mean search times involved in comparison classification and location of target symbols will be obtained by fitting the linear function of the form $y_{c}=a+b x$ as described earlier. The slope of the line of best fit i.e. the coefficient ' $b$ ' of the fitted function will provide the required mean discrimination time per symbol. The ' $y$ ' intercepts or the constant ' $a$ ' of the fitted function is theoretically located at a point where focal attentive processes involved in comparison, classification and location of target symbols have not started operating. It represents the condition where the deletion or absence of a stage is obtained by mathematical extrapolation. The intercept provides an estimate of the sum of time taken for segmentation of the target area from the rest of the prototype map's focal realm, and preattentive processing of the visual information from this area. This time will also include time spent on other involved processing steps of encoding and processing the standard circle symbol.

\section{VII.3 Test Results}

The time spent on segmentation of target area, encoding and processing of the anchor or a standard proportional symbol and response organisation along with the motor response and other extraneous operations is likely to be almost equal for target areas of a prototype map or target areas on different prototype maps. Any variation in the value of the ' $y$ ' intercept will therefore represent the variation in the time taken in the preattentive processing of information These variations, then, will provide a measure of the effect of selected independent variables i.e. uniform neutral gray fill as well the graded value fills of neutral gray on the efficiency of symbol discrimination from the open and gray area filled proportional circle maps.

The accuracy in the visual search results will be determined by comparing the obtained responses with the symbol size information available from the prototype maps. The errors will be measured as the number of wrongly identified symbols i.e. larger or smaller circles identified as circles of the given size as well the number of circle or square symbols of the given size not identified as such in a given area and will be expressed as percentage of the total number of these symbols of the selected size.

The results of visual search experiment used to test the selected hypothesis that the use of gray area fills both uniform and graded has increased the processing efficiency of different levels of preattentive processing i.e. segmentation of different map components, segregation of input in the dimension of depth. The processing efficiency of this stage will be reflected in the ease of these operations i.e. the time spent on processing. Tables 2 and 3 show an overall improvement in preattentive processing time in case of the maps symbolised with gray area fills as compared to maps conventionally symbolised with open proportional circles and squares. The increased dimensionality of the symbol forms obtained through the use of gray area fills both uniform and graded in structuring the map symbols appears to have overcome the limitations of the open circle and square symbol which basically relates to the inadequacies of the open graphic form of symbols.

These results can be seen as an improvement in the functional effectiveness of these maps due to the incorporation of both uniform gray and graded area fills in the symbol design together with the design improvements done in the legend design. These area fills appear to have controlled interference in the preattentive visual information processing from the maps to appreciable extents. This is seems to be made possible by (1) a reduction of noise in the visual input from the maps caused by the open symbol form used conventionally on proportionally symbolised circle and square maps and (2) due to the availability of additional 
visual characteristics from the area fills introduced in the symbol design The results in Tables 2 and 3 showing an improved preattentive processing time between maps of open and grey filled circle and square symbolised maps can be attributed to changes in the surface character of the of the circle and square symbols relating to the appearance of the symbol form and use of graded gray fills. The changes in surface character have created a primary heterogeneity in the map image in its focal realm. It has resulted in a primitive segmentation between these symbols and the elements of locational component and the homogeneous white plane of the substrate in the focal realms. The manner, in which the change in surface character is brought about, creates a visual barrier or resistance for the eyes to penetrate. This visual resistance, in a way, provides areal extent as well
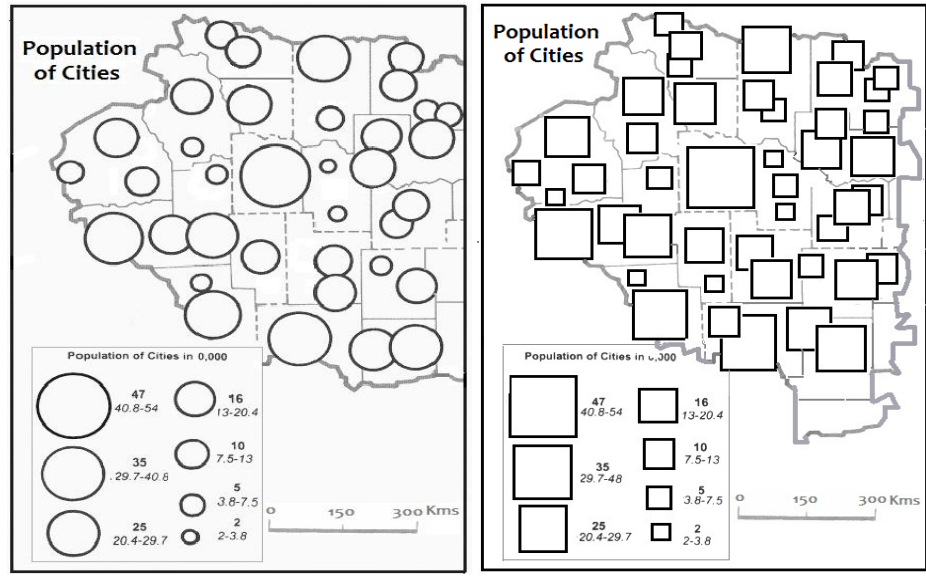

Figure 9. The prototype maps used in the experiments

micro-structure to the circle and square symbols (Katz. 1958 [47], Koffka 1935[48]). In addition, it is expected to give, in Rubin's (1958) [22] words, a 'thing character' to hitherto undifferentiated open circle forms. The information in the symbol forms, so far limited to contours only, thus appears to have been be reinforced in the very early phases of the process of segmentation.

Table 2. Results of Pre-attentive time spent during proportional circle discrimination

\begin{tabular}{c|c|c}
\hline Area Fills & $\begin{array}{c}\text { Preattentive } \\
\text { Processing } \\
\text { Time } \\
\text { (y intercept 'a') } \\
\text { sec/m. sec }\end{array}$ & $\begin{array}{c}\text { Percent } \\
\text { Improvement } \\
\text { In processing } \\
\text { time }\end{array}$ \\
\hline $\begin{array}{c}\text { Open } \\
\text { Circles }\end{array}$ & 2.490 & \\
\hline \hline $\begin{array}{l}\text { Uniform Gray } \\
\text { Filled Circles }\end{array}$ & 2.290 & 08.03 \\
\hline \hline $\begin{array}{l}\text { Graded Gray } \\
\text { Filled Circles }\end{array}$ & 1.792 & 28.03 \\
\hline
\end{tabular}

These results also have support in the experimental evidence obtained by Wever (1927: 217-218) [49] which shows that a large difference in luminous reflectance between a relatively dark homogeneous figure

Table. 3 Results of Pre-attentive time spent during proportional square discrimination

\begin{tabular}{c|c|c}
\hline Area Fills & $\begin{array}{c}\text { Preattentive } \\
\text { Processing } \\
\text { Time } \\
\text { (y intercept 'a') } \\
\text { sec/m. sec }\end{array}$ & $\begin{array}{c}\text { Percent } \\
\text { Improvement } \\
\text { In processing } \\
\text { time }\end{array}$ \\
\hline $\begin{array}{c}\text { Open } \\
\text { Squares }\end{array}$ & 2.891 & 0 \\
\hline \hline $\begin{array}{c}\text { Uniform Gray } \\
\text { Filled Squares }\end{array}$ & 2.618 & 09.44 \\
\hline \hline Graded Gray & 2.260 & 22.2 \\
\hline Filled Squares & & 2 \\
\hline
\end{tabular}


having a surface character and an equally homogeneous white ground is required for a 'perfect' figure-ground experience and it is in addition to the requirement of a sharp and continuous contour. Oyama (1960) [50] too has come to a similar conclusion for the necessity of a large value contrast between the figure and ground for the stabilisation of figure. Lindauer and Lindauer (1970) [51], using the Rubin's face and vase image with a control stimulus of only the black outline, have also shown that the number of responses for the filled area as figure had increased with the increase in the contrast between the two areas. In case of maps similar conclusions were drawn by Wood (1968) [38] and later by Spiess (1978) [52] that the segmentation of different shapes as figures is mainly a function of the degree of contrast in the two fields of the map. McCleary's (1981) [53] results similarly lay stress on the importance of the role of value variations in creating heterogeneity and articulation in the different visual fields of a map for the separation of different shapes as figures. The stability in the segmented figures has similarly been noticed to be further strengthened by the surface texture created homogeneity in the figural areas. Wever (1927: 217, 223) [49] found that the surface texture provides a difference in the depth localisation of figure and ground. Dent (1972:88) [21] too has experimentally demonstrated the importance of the role of texture differences in the development and localisation of figure and ground surfaces in maps. Such results were also obtained in a relatively recent study in cartography related to this stage in information processing from thematic maps ( McCleary 1981) [53].

The chosen mode of the application of the uniform and graded gray area fills to the black outlines of circles and squares lends a microstructure to these symbols. This obtained surface character, in addition, results in producing a kind of internal unity in the plan forms of the circle symbols. The unity provides information on areal extents of the circles and is expected to produce an improvement in the quality of circle size information made available to the earliest processes of the visual information processing system (Wood 1972) [11].

The method used for symbol definition and construction has also controlled another form of interference in the visual information processing by removing certain insufficiencies in the legend. In addition, the improvement in the legend design is seen to remove some other deficiencies by ensuring the availability of adequate number as well appropriate sizes for standard symbols for use in the legend (Table 4).

The graded gray area fills supplement the circle size information by providing better discriminability in the size ranges where larger JNDs are required for discrimination. In this case of the supplementing role of 'value' incorporated in graded gray fills in increasing the discriminability size information reliance can be placed on the empirical discrimination functions derived by Munsell (1991) [54].This additional information seems to have overcome the limitations of areal size based discriminability of proportional circle and square symbols.

Table 4. Results of experiment of discrimination on proportional square symbolised maps

\begin{tabular}{|c|c|c|c|c|c|c|}
\hline Area Fills & $\begin{array}{c}\text { Average } \\
\text { Processing } \\
\text { Speed of } \\
\text { Discrimination } \\
\text { Seconds/mi.sec }\end{array}$ & $\begin{array}{l}\text { \%Improvement } \\
\text { In Speed }\end{array}$ & $\begin{array}{c}\% \\
\text { Error }\end{array}$ & $\begin{array}{c}\% \\
\text { Improvement } \\
\text { In Error }\end{array}$ & Sest. & $\begin{array}{c}\text { Degree of } \\
\text { Fit of } \\
\text { Estimating } \\
\text { Function } \\
\left(r^{2)}\right.\end{array}$ \\
\hline Open Squares & 4.440 & & 10 & & 0.887 & 0.984 \\
\hline \multirow[t]{2}{*}{$\begin{array}{l}\text { Uniform Gray } \\
\text { Filled Squares }\end{array}$} & 3.856 & & 09 & & 1.113 & 0.976 \\
\hline & & 13.0 & & 10 & & \\
\hline \multirow[t]{2}{*}{$\begin{array}{l}\text { Graded Gray } \\
\text { Filled Squares }\end{array}$} & 3.480 & & 08 & & 1.542 & 0.944 \\
\hline & & 22.0 & & 20 & & \\
\hline
\end{tabular}

Experimental evidence in support of this increased discriminability can be seen in the early work of Erikson and Hake (1955) [55], observations of Arnheim (1969: 323) [31] and Mersey's (1990) [56] work in cartography. The role of value in selection and grouping may similarly be seen in adding to the size dependent differentiation and selectivity leading to efficient chunking of the segregated input. This becomes possible due to the availability of faster information on the dimension of value, which leads to better symbol definitions. When combined with size this selectivity is further increased because of the human system's quick response to combined changes in these two dimensions (Table 5). 
In addition, the additional gradients of value appear to have made up for the absence of the gradients produced by the obliquity in the relative locations of the symbols in graduated circle maps. In the current context the gradients of value have been found to be most effective in engendering the relations of order in the three dimensional space (Mersey 1990 [56], Egusa 1983 [57], Cuff 1973a [58], 1974a [59], 1974b [60]).

The quality of specificity and memorability provided by graded gray steps in the legend symbols is further seen to ensure an efficient probe and test sequence in top-down verifications. It is at the same time also appears to prevent a continuous shift of attention from map to legend and back which affects the efficiency of information processing at this stage (DeLucia and Hiller 1982: 48) [34]. The availability of adequate number of standard symbols of appropriate size when combined with the specificity and the role of memorability in selection as the required anchors have resulted in an effective magnitude based discrimination in case of circle symbolised maps. The support for the results are seen in the works of Stevens (1975: 26) [16] and Baird (1970)

Table 5. Results of experiment of discrimination on proportional circle symbolised maps

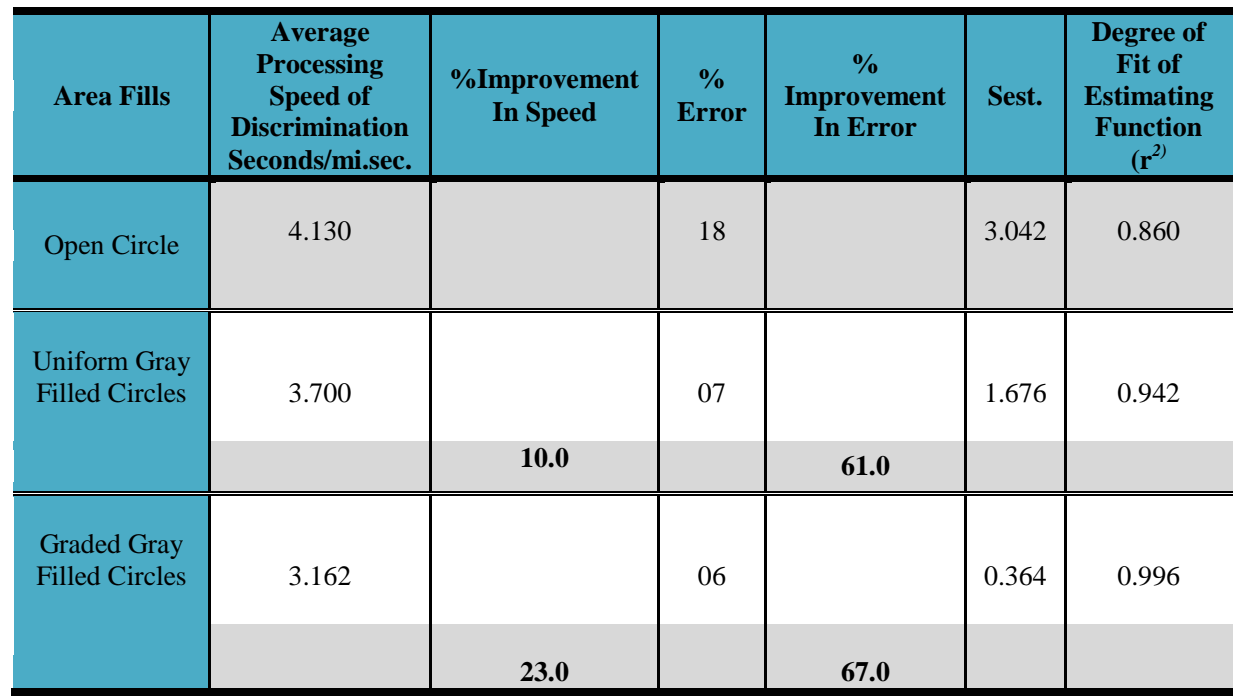

[14] along with the original work of Helson (1953) and its extension to magnitude based discrimination tasks from proportional circle and squares symbolised maps in the works of by Cox (1973 [17], 1976[18]).

\section{CONCLUSIONS}

The use of uniform and graded gray fills in design and structuring of proportional circle and square symbols was made in configural redundant manner to find out how this design change can bring about an improvement in the functional effectiveness of maps leading to a better comprehension of these maps. The total effect in subjects' performance was seen as an enhancement in the functional effectiveness of these maps caused due to the symbol design improvements. The symbol form deficiency of open symbol forms in both cases of circle and square symbols was removed by both uniform and graded area fills in the symbol outline forms. However the graded area fills in both types of symbols have shown better results due to the added effect of the modulations of the graphic variable of value with the concurrent alterations in the graphic variable of size. These improvements have been complemented by the simultaneous changes in legend design. Legend design effects are better in case of graded area fills due to production of specificity and property of standard symbols in the legend.

\section{REFERENCES}

[1] Tobler, W.R. (1973) Choropleth Maps without Class Intervals?, Geographical Analysis, 5, 262-265.

[2] Brassel, K.E., Utano, J.J. (1979) Design Strategies for Continuous-Tone Area Mapping. American Cartographer, 6 , pp.39-50

[3] Muller, Jean-Claude (1979) Perception of Continuously Shaded Maps, Annals, Association of American Geographer, 69:2,240-249

[4] Peterson, M.P. (1979) An Evaluation of Unclassed Crossed-Line Choropleth Mapping, American Cartographer, 6, 21-37.

[5] Haber, R. N. and Hershenson, M. (1973) The Psychology of Visual Perception Holt, Rinehart \& Winston, New York.

[6] Wright, John K. (1944) A Proposed Atlas of Diseases, Geographical Review, 34, 642-652.

[7] Robinson, A. H. (1955) The 1837 Maps of Henrey Dury Harness, Geographical Journal, 121, 440-452. 
[8] Flannery, J. J. (1956) The Graduated circle: A Description Analysis and Evaluation of a Quantitative Symbol, Unpublished Ph. d. Dissertation, University of Wisconsin.

[9] Akhtar, M. (1990) An Analysis and Evaluation of Dot Maps as spatial information Display System -A Study in Theoretical Thematic Cartography, Unpublished Ph.D. Thesis, University of Patna, Patna.

[10] William, B. L. (1958) Map Symbols: Equal-Appearing Intervals for Printed Screens, Ann. Assoc. of Am. Geog. 48, pp. 132-139.

[11] Wood, M. (1972) Human Factors in Cartographic Communication, Cartographic Journal, 9, 123-132.

[12] John, V. (2003) The Observed Inadequacies in the Design of Graduated Circle Symbolised Maps, National Geographer, 38:1, pp. 1-16

[13] Meihoefer, Hans-Joachim (1969) The Use of Circle in Thematic Maps A Study in Visual Perception of a Cartographic Symbol, Unpubl. Ph. D. Dissertation, University of Washington

[14] Baird J. C. (1970) The Psychophysical Analysis of Visual Space, Pergamon Press, Oxford.

[15] Dobson, M.W.(1974) Refining Legend Values for Proportional Circle Maps, Canadian Cartographer, 11:1, 45-53.

[16] Stevens, S. S. (1975) Psychophysics: Introduction to its Perceptual, Neural and Social Aspects, Stevens G (ed.) Wiley, New York.

[17] Cox, C. W. (1973) Adaptation-Level Theory as an Aid to Understanding of Map Perception, Proceedings American Congress Surveying and Mapping, 33rd Annual Meeting, 334-359.

[18] Cox, Carleton W. (1976) Anchor Effects and the Estimation of Graduated Circles and Squares, Cartography and Geographic Information Science, 31, pp. 65-74

[19] Chang, K. T. (1980) Circle Size Judgment and Map Design, American Cartographer, 7:2, 155-162

[20] Posner and Rogers (1978) Chronometric Analysis of Abstraction and Recognition in Handbook of Learning and cognitive Processes, (Ed.) W.K. Estes, Lawrence Erblaum Publishers, Hillsdale, 143-188.

[21] Dent, B. D. (1972) Visual Organization and Thematic Map Communication, Annals, Association of American Geographer, 62, 79-93.

[22] Rubin, E. (1958) Figure and Ground, in Readings in Perception, D. C. Beardslee and M. Wertheimer (eds.) Von Nostrand, Princeton

[23] Schlichtman, H. (1979) Codes in Map Communication, Canadian Cartographer.16, 81-97.

[24] Schlichtman, H. (1985) Characteristic Traits of the Semiotic System Map Symbolism Cartographic Journal, 22, 2330 .

[25] Neisser, U. (1967) Cognitive Psychology, Appleton Century-Crofts, New York

[26] Treisman, A. \& Gelade, G. (1980).A feature-integration theory of attention. Cognitive Psychol. 12, pp.97-136

[27] Wolfe, J. M. (1994) Guided search 2.0: A Revised Model of Visual Search. Psychon. Bull. Rev. 1, 202-238

[28] Palmer, S. E. (1975) Visual Perception and World Knowledge: Notes on a Model of Sensory-cognitive Interaction, in Explorations in Cognition, D. A. Norman, D. E. Rumelhart (ed.) San Francisco: W.H. Freeman, 279-307

[29] Eastman, J. R. (1985) Cognitive Models and Cartographic Design Research, Cartographic Journal, 22:2, 95-101.

[30] Navon, D. (1977) Forest before Trees: The Precedence of Global Features in Visual Perception, Cognitive Psychology, 9:3, 353-383.

[31] Arnheim R. (1969) Art and Visual Perception 'a psychology of the creative eye' Faber and Faber, London.

[32] Gibson J. J. (1950) The Perception of the Visual World, Houghten Mifflin Co. Boston.

[33] Robinson, A. H., Randall D. Sale, R. D., Morrison, J. and Muehrcke, Phillip, C. (1995) Element of Cartography, (VI ${ }^{\text {th }}$ Eds.) John Wiley, New York.

[34] DeLucia, A. A., and Hiller. W. (1982) Natural Legend Design for Thematic Maps, Cartographic Journal, 19:1, 4652.

[35] Chang, K. T. (1969) A psychophysical study of quantitative map symbols, Unpublished Master's Thesis, Clark University

[36] Cox, C. W.(1975) Psychophysical Research and Map Reading Analysis, Seminar on Map Reading and Perception, Proceedings of the Autocarto-2 pp. 233-37

[37] Helson, Harry (1953) The Theory of Adaptation Level, Reprinted in Reading in Perception, D. C. Beardslee and M. Wertheimer, eds., (1958). Von Nostrand, Princeton

[38] Wood, M. (1968) Visual Perception and Map Design, Cartographic Journal, 5, 54-64

[39] Cuff, D. J. and Mattson M.T. (1984) Thematic Maps: Their Design and Production. Methuen New York, London

[40] Castner, H. W. (1983) Research Questions and Cartographic Design, in Graphic Communication and Design in Contemporary Cartography, D. R. F. Taylor (eds.) pp. 87-113, John Wiley, New York,

[41] Donders F.C. (1868) On the Speed of Mental Processes, Translated in 1969 by W. G. Koster, Acta Psychologica, 30 , 412-431

[42] Neisser, U. (1963) Decision Time without Reaction Time: Experiments in Visual Scanning, Amer. Jour. of Psychology, 76, 376-385.

[43] Neisser, U. (1964) Visual Search, Scientific American, 210, 94-103.

[44] Sternberg S. (1966) High Speed Scanning in Human Memory, Science, 153, 652-654.

[45] Sternberg S. (1967) Two Operations in character-Recognition: Some Evidence from Reaction-Time Measurements, Perception and Psychophysics, 2, 45-53.

[46] Sternberg S. (1969) The Discovery of Processing Stages: Extensions of Donders' Method, in Attention and Performance II, W.G. Koster (Ed.), Acta Psychologica, 30, 276-315.

[47] Katz, D. (1958) The Modes of Appearance of colours, in Readings in Perception. D. C. Beardslee and M. Wertheimer (eds.)Von Nostrand, Princeton. 
[48] Koffka, K. (1935) Points and Lines as Stimuli, in Readings in perception, D.C.Beardslee and M. Wertheimer (eds.) (1958), Von Nostrand, Princeton.

[49] Wever, E. (1927) Figure and Ground in the Visual Perception of Form, 38, 194-226 American Journal of Psychology, 38, 194-226.

[50] Oyama, T. (1960) Figure-Ground Dominance as a Function of Sector Angle, Brightness Hue and Orientation, Journal of Experimental Psychology, 60:5, 299-305.

[51] Lindauer, M., Lindauer, J.(1970) Brightness Differences and the perception of Figure Ground Journal of Experimental Psychology, 84:2, 291-95.

[52] Spiess E. (1978) Some Graphic Means to Establish Visual Levels in Map Design, International Cartographic Association.

[53] McCleary, G. (1981) How to Design an Effective Graphics Presentation, Harvard Library of Computer Graphics, 1981 Mapping Collection, 17, P.A. Moore (ed.) 15-64.

[54] Munsell, A. H. (1991) A Colour Notation 17 ed., Munsell Colour, Baltimore.

[55] Eriksen, C. W. and Hake, H. W. (1955) Multidimensional Stimulus Differences and Accuracy of Discrimination, Journal of Experimental Psychology, 50, 153-160.

[56] Mersey, Janet E. (1990) Colour and Thematic Map Design: The Role of Colour Scheme and Map Complexity in Choropleth Map Communication, Cartographica, 27:3

[57] Egusa, H. (1983) Effects of Brightness, Hue and Saturation on Perceived Depth between Adjacent regions in the Visual Field, Perception, 12:2, 167-175.

[58] Cuff, D. J. (1973a) Shading on Choropleth Maps: Some Suspicions Confirmed. Proceedings, Association of American Geographer 5, 50-54.

[59] Cuff, D. J. (1974a) Perception of Colour Sequences on Maps of Atmospheric Pressure Professional Geographer, 26:2, 166-171.

[60] Cuff, D. J. (1974b) Impending Conflict in Color Guidelines for Maps of Statistical Surfaces Canadian Cartographer, $11: 1,54-58$. 